\title{
Relationship of Salivary Occult Blood With General and Oral Health Status in Employees of a Japanese Department Store
}

\author{
Kazushi Segawa ${ }^{a}$, Hideo Shigeishib, c, Munehito Fujii ${ }^{a}$, Kazuki Noumia ${ }^{a}$, Fuminori Yamanaka ${ }^{a}$, \\ Katsumi Kamikawa ${ }^{a}$, Shinsuke Arakawa ${ }^{a}$, Masaru Sugiyama ${ }^{b}$
}

\begin{abstract}
Background: The Japanese Ministry of Health started screening for periodontal disease as part of senior health services in 1995. However, only a small number of workplaces conduct regular dental checkups in Japan. Therefore, the prevalence of periodontal disease and other oral health conditions has not been fully determined in workers in Japan. This study aimed to clarify the usefulness of a salivary occult blood test (SOBT) to assess periodontal disease, and to investigate the association of salivary occult blood with general and oral health in Japanese employees.
\end{abstract}

Methods: A cross-sectional study was conducted among department store workers in Hiroshima city. Subjects were 449 workers who received regular health checkups including dental examinations in 2018. An SOBT using monoclonal antibody against human hemoglobin was performed. Periodontal status was evaluated using the Community Periodontal Index (CPI). We investigated the association of salivary occult blood with general and oral health in 338 employees ( 85 men, 253 women; mean age 41.4 years, range 19 - 69 years).

Results: Univariate analysis revealed a significant relationship between salivary occult blood and sex, body mass index (BMI), diabetes, CPI, daily brushing frequency, and number of untreated teeth $(\mathrm{P}$ $=0.034, \mathrm{P}=0.003, \mathrm{P}=0.022, \mathrm{P}=0.007, \mathrm{P}=0.004$, and $\mathrm{P}=0.015$, respectively). Furthermore, BMI, diabetes, CPI, and brushing frequency were significantly associated with salivary occult blood in binomial logistic regression analysis (odds ratio 1.09, $\mathrm{P}=0.014$; odds ratio 9.38, $\mathrm{P}=0.047$; odds ratio $1.31, \mathrm{P}=0.004$; and odds ratio 0.70 , $\mathrm{P}=0.045$, respectively). These results suggest that positivity in the SOBT is importantly associated with periodontal disease and diabetes. Interestingly, subjects aged $\geq 35$ years with metabolic syndrome

Manuscript submitted November 24, 2018, accepted December 20, 2018

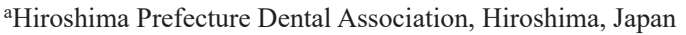

${ }^{b}$ Department of Public Oral Health, Program of Oral Health Sciences, Graduate School of Biomedical \& Health Sciences, Hiroshima University, Japan

${ }^{\mathrm{c} C o r r e s p o n d i n g ~ A u t h o r: ~ H i d e o ~ S h i g e i s h i, ~ D e p a r t m e n t ~ o f ~ P u b l i c ~ O r a l ~ H e a l t h, ~}$ Program of Oral Health Sciences, Graduate School of Biomedical \& Health Sciences, Hiroshima University, 1-2-3 Kasumi, Minami-ku, Hiroshima 7348553, Japan. Email: shige@hiroshima-u.ac.jp

doi: https://doi.org/10.14740/jocmr3702 exhibited a significantly higher positive rate of salivary occult blood than those without metabolic syndrome $(\mathrm{P}=0.01)$.

Conclusions: The SOBT was a reliable screening method for periodontal disease, and positivity in the test was related to diabetes in Japanese employees. Further examinations are required to clarify the association of salivary occult blood with other systemic diseases.

Keywords: Salivary occult blood test; Periodontal disease; Metabolic syndrome; Workers

\section{Introduction}

The World Health Organization has reported that noncommunicable diseases such as cancer, cardiovascular disease, chronic respiratory disease and diabetes are major causes of death in people over 30 years old, and accounted for over $60 \%$ of deaths worldwide in 2010 [1, 2]. Periodontal disease is a common oral lesion that is prevalent in people aged over 40, and is closely associated with noncommunicable diseases (e.g. cardiovascular disease and diabetes) [3-6].

A survey on dental diseases conducted by the Ministry of Health, Labour and Welfare in Japan revealed that the percentage of people with periodontal pockets $\geq 4 \mathrm{~mm}$ was higher in 2016 than in 2011 in all age groups [3]. Additionally, people aged $65-74$ recorded the highest rate of periodontal pockets $\geq$ $4 \mathrm{~mm}$ [3]. These statistics suggest that periodontal disease is a vital health problem among Japanese people.

The Japanese Ministry of Health started screening for periodontal disease as part of senior health services in 1995 . In 2005, people aged in their 60s and 70s were newly targeted for this screening as well as those aged in their $40 \mathrm{~s}$ and $50 \mathrm{~s}$. However, only a small number of workplaces conduct regular dental checkups, because dental checkups are not a mandatory component of occupational health services in Japan. Therefore, the prevalence of periodontal disease and other oral health conditions has not been fully determined in workers in Japan.

In 2015, Hiroshima Prefectural Dental Association started conducting dental checkups for employees of a department store in Hiroshima city. The aims of these checkups were to 
detect oral lesions early, to recommend early visits to a dental clinic, and to give appropriate health guidance to maintain the health of employees. Additionally, we investigated hemoglobin positivity using a salivary occult blood test (SOBT) to screen for periodontal disease. An SOBT using monoclonal antibody against human hemoglobin has been used in investigating periodontal health, and it may be useful as a screening method for periodontal disease $[7,8]$. An SOBT using antihuman hemoglobin monoclonal antibody has been used to reliably assess periodontal inflammation in pregnant women [7].

The objectives of this study were to clarify the usefulness of an SOBT using monoclonal antibody against human hemoglobin to assess periodontal disease, to investigate the association between the SOBT and general and oral health, and finally to determine the correlation between metabolic syndrome and factors associated with oral health in Japanese employees.

\section{Materials and Methods}

\section{Subjects}

A cross-sectional study was conducted among workers of a large-scale retail store in Hiroshima city. Subjects were 449 workers who received regular health checkups, including a dental examination, in 2018. Importantly, individuals who ate or brushed their teeth within $2 \mathrm{~h}$ of the SOBT were excluded from the study to omit positive cases caused by eating or brushing. The final number of subjects enrolled in this study was 338 ( 85 men, 253 women; mean age 41.4 years, range 19 - 69 years). The study design was approved by the Ethical Committee of Hiroshima University (Permission no. E-1125), and all participants signed an informed consent agreement.

\section{Data collection from regular health checkup}

We collected the results of the health examination (i.e. sex, age, body mass index (BMI), waist circumference, blood pressure, and blood examination such as fasting blood sugar (FBS), triglycerides (TGs), and high-density lipoprotein (HDL) cholesterol). Waist circumference, FBS, TG and HDL cholesterol were examined in subjects aged 35 years and older. Metabolic syndrome was diagnosed when subjects had a waist circumference $\geq 85 \mathrm{~cm}$ for men and $\geq 90 \mathrm{~cm}$ for women, and two or more of the following: abnormal lipid metabolism ( $\mathrm{TG} \geq 150$ $\mathrm{mg} / \mathrm{dL}$ and/or HDL cholesterol $<40 \mathrm{mg} / \mathrm{dL}$ ), high blood pressure (systolic blood pressure $\geq 130 \mathrm{~mm} \mathrm{Hg}$ and/or diastolic blood pressure $\geq 85 \mathrm{~mm} \mathrm{Hg}$ ), or abnormal glucose metabolism (FBS $\geq 110 \mathrm{mg} / \mathrm{dL})$ [9].

\section{SOBT}

Prior to the oral examination, an SOBT using monoclonal antibody against human hemoglobin (Perioscreen; Sunstar, Osaka, Japan) was performed according to the manufacturer's protocol. Briefly, participants rinsed with $3 \mathrm{~mL}$ distilled water for 10 $\mathrm{s}$ and then expectorated into a paper cup. A strip was immersed into the rinse sample and the colored band was checked after 5 min.

\section{Oral examination}

The oral examination including a periodontal examination (i.e. assessment of probing depth and bleeding on probing) was performed by dentists of the Hiroshima Prefecture Dental Association. Periodontal condition was assessed according to the Community Periodontal Index (CPI) [10]. The CPI code consists of the following five categories: healthy (code 0 ), gingival bleeding (code 1 ), calculus (code 2 ), shallow periodontal pockets of 4 - $5 \mathrm{~mm}$ (code 3 ), and deep periodontal pockets of $6 \mathrm{~mm}$ or deeper (code 4) [11]. Additionally, the number of treated teeth, untreated teeth and all existing teeth was recorded.

\section{Data collection using a questionnaire}

A questionnaire was administered containing questions about medical history, smoking history, and oral health habits such as daily brushing frequency and the use of additional tooth cleaning appliances besides a toothbrush (i.e. dental floss and interdental brush).

\section{Statistical analysis}

Statistical analysis was performed using SPSS software, version 24.0 (IBM Corp., Armonk, NY, USA). A Chi-squared test or Fisher's exact test and Mann-Whitney U test were used to evaluate significant differences between salivary occult blood and clinical factors. Adjusted standardized residuals greater than 1.96 in the Chi-square test indicated that the number of cases was significantly larger than expected. P values less than 0.05 were regarded as statistically significant. Binomial logistic regression analysis was conducted using salivary occult blood as the dependent variable, and variables with a $\mathrm{P}$ value of $<0.20$ through univariate analysis as independent variables. The Hosmer-Lemeshow test was used to evaluate the goodness of fit of the logistic regression models, indicating a good fit of the model if the significance value was $>0.05$.

\section{Results}

\section{Correlation between salivary occult blood and factors as- sociated with general and oral health}

We examined the relationship between salivary occult blood and factors associated with general and oral health (Table 1). Univariate analysis revealed that there was a significant correlation between salivary occult blood and sex $(\mathrm{P}=0.034$, Chi-squared test). BMI was significantly higher in the salivary occult blood-positive group than in the negative group $(\mathrm{P}=$ 0.003, Mann-Whitney U test). Additionally, subjects with dia- 
Table 1. Correlation Between Salivary Occult Blood and Factors Associated With General and Oral Health

\begin{tabular}{|c|c|c|c|}
\hline & \multicolumn{2}{|c|}{ SOBT } & \multirow{2}{*}{ P value } \\
\hline & Negative & Positive & \\
\hline \multicolumn{4}{|l|}{ Sex } \\
\hline Men (85) & $47(55.3 \%)$ & $38(44.7 \%)$ & 0.034 \\
\hline Women (253) & $172(68.0 \%)$ & $81(32.0 \%)$ & \\
\hline Age & $40.6 \pm 13.7$ & $43.0 \pm 14.1$ & 0.119 \\
\hline BMI & $21.6 \pm 3.1$ & $23.0 \pm 4.0$ & 0.003 \\
\hline $\mathrm{SBP} \geq 130 \mathrm{~mm} \mathrm{Hg}$ and/or $\mathrm{DBP} \geq 85 \mathrm{~mm} \mathrm{Hg}(81)$ & $46(56.8 \%)$ & $35(43.2 \%)$ & \\
\hline \multicolumn{4}{|l|}{ Smoking } \\
\hline Never smokers (242) & $159(65.7 \%)$ & $83(34.3 \%)$ & 0.856 \\
\hline Former smokers $(58)$ & $36(62.1 \%)$ & $22(37.9 \%)$ & \\
\hline \multicolumn{4}{|l|}{ Hypertension } \\
\hline$(-)(314)$ & $203(64.6 \%)$ & $111(35.4 \%)$ & 0.842 \\
\hline$(+)(24)$ & $16(66.7 \%)$ & $8(33.3 \%)$ & \\
\hline \multicolumn{4}{|l|}{ Diabetes } \\
\hline$(-)(332)$ & $218(65.7 \%)$ & $114(34.3 \%)$ & 0.022 \\
\hline$(+)(6)$ & $1(16.7 \%)$ & $5(83.3 \%)$ & \\
\hline \multicolumn{4}{|l|}{ Hyperlipidemia } \\
\hline$(-)(322)$ & $211(65.5 \%)$ & $111(34.5 \%)$ & 0.204 \\
\hline$(+)(16)$ & $8(50.0 \%)$ & $8(50.0 \%)$ & \\
\hline \multicolumn{4}{|l|}{ Cardiovascular disease } \\
\hline Code $3(50)$ & $26(52.0 \%)$ & $24(48.0 \%)$ & \\
\hline Code $4(32)$ & $17(53.1 \%)$ & $15(46.9 \%)$ & \\
\hline Number of untreated teeth & $0.8 \pm 1.5$ & $1.3 \pm 2.1$ & 0.015 \\
\hline Number of treated teeth & $8.7 \pm 6.3$ & $8.9 \pm 6.2$ & 0.706 \\
\hline Number of remaining teeth & $27.9 \pm 2.4$ & $27.3 \pm 3.7$ & 0.136 \\
\hline \multicolumn{4}{|l|}{ Daily brushing frequency } \\
\hline Once a day (31) & $13(41.9 \%)$ & $18(58.1 \%)$ & 0.004 \\
\hline Twice a day (116) & $74(63.8 \%)$ & $42(36.2 \%)$ & \\
\hline Three times a day (189) & $132(69.8 \%)$ & $57(30.2 \%)$ & \\
\hline Not every day (2) & $0(0.0 \%)$ & $2(100 \%)$ & \\
\hline \multicolumn{4}{|l|}{ Dental flossing and interdental brushing } \\
\hline Dental flossing (74) & $46(62.2 \%)$ & $28(37.8 \%)$ & 0.080 \\
\hline Interdental brushing (70) & $52(74.3 \%)$ & $18(25.7 \%)$ & \\
\hline Both $(27)$ & $21(77.8 \%)$ & $6(22.2 \%)$ & \\
\hline Never (167) & $100(59.9 \%)$ & $67(40.1 \%)$ & \\
\hline
\end{tabular}

Mann-Whitney U-test, Chi-squared test or Fisher's exact test were used for statistical analysis. P values less than 0.05 were regarded to be statistically significant. 
Table 2. Logistic Regression Analysis With Salivary Occult Blood as Dependent Variable

\begin{tabular}{llll}
\hline Variables & Odds ratio & 95\% confidence interval & Pvalue \\
\hline BMI & 1.09 & $1.02-1.17$ & 0.014 \\
Diabetes & 9.38 & $1.03-85.55$ & 0.047 \\
CPI & 1.31 & $1.09-1.57$ & 0.004 \\
Daily brushing frequency & 0.70 & $0.49-0.99$ & 0.045 \\
\hline
\end{tabular}

Binomial logistic regression analysis was conducted using "salivary occult blood" as the dependent variable. P values less than 0.05 were regarded to be statistically significant.

betes recorded a higher positive percentage of salivary occult blood $(83.3 \%)$ than those without diabetes $(34.3 \%)$. There was a significant positive correlation between salivary occult blood and diabetes $(P=0.022$, Fisher's exact test $)$. A significant correlation between salivary occult blood and the CPI code was observed ( $\mathrm{P}=0.007$, Chi-squared test). Subjects with a CPI code of 0 recorded a significantly lower positive rate of salivary occult blood. Significant associations were found between salivary occult blood and the number of untreated teeth and daily brushing frequency $(\mathrm{P}=0.015$, Mann-Whitney $\mathrm{U}$ test; $\mathrm{P}$ $=0.004$, Fisher's exact test, respectively). Subjects brushing three times a day recorded a significantly lower positive rate of salivary occult blood.

To examine the strength of the correlation between salivary occult blood and clinical factors, binomial logistic regression analysis was performed using salivary occult blood as the dependent variable, and variables showing a $\mathrm{P}$ value of $<$ 0.20 such as sex, age, BMI, hypertension, diabetes, CPI, dental flossing and interdental brushing, number of untreated teeth, and number of remaining teeth as independent variables. The $\mathrm{P}$ value for the Hosmer-Lemeshow test was 0.758 , indicating a good fit of the model. BMI, diabetes, CPI, and daily brushing frequency were significantly associated with salivary occult blood (odds ratio 1.09, $\mathrm{P}=0.014$; odds ratio 9.38, $\mathrm{P}=0.047$; odds ratio $1.31, \mathrm{P}=0.004$; and odds ratio $0.70, \mathrm{P}=0.045$, respectively) (Table 2).

\section{Correlation between salivary occult blood and factors as- sociated with general and oral health in workers aged $\geq 35$ years}

We examined the correlation between salivary occult blood and factors associated with general and oral health in subjects aged $\geq 35$ years (Table 3 ). Subjects with a high level of TGs or high FBS exhibited a greater positive rate of salivary occult blood $(77.8 \%$ and $59.1 \%$, respectively) than those with low level TGs or FBS (31.5\% and 32.8\%, respectively). Statistically significant differences were observed in TGs, FBS, metabolic syndrome, diabetes, daily brushing frequency, and number of untreated teeth (Table 3). Importantly, subjects brushing their teeth three times a day showed a significantly lower positive rate of salivary occult blood.

Binomial logistic regression analysis was performed using age, BMI, blood pressure, hypertension, TGs, HDL cholesterol, FBS, metabolic syndrome, diabetes, CPI, brushing frequency, dental flossing and interdental brushing, number of untreated teeth, and number of remaining teeth as independent variables. P values for the Hosmer-Lemeshow test were 0.149, indicating a good fit of the model. TGs and CPI were significantly associated with salivary occult blood (odds ratio 7.59, $\mathrm{P}$ $<0.001$; odds ratio $1.40, \mathrm{P}=0.007$, respectively) (Table 4). In this model, TG was the most significant component of metabolic syndrome associated with salivary occult blood.

\section{Correlation between metabolic syndrome and factors as- sociated with oral health}

Finally, we examined the relationship between metabolic syndrome and the results of the oral examination in subjects aged $\geq 35$ years. Salivary occult blood-positive subjects were more likely to exhibit metabolic syndrome $(13.9 \%)$ than negative subjects (3.9\%). Significant associations of metabolic syndrome with salivary occult blood and daily brushing frequency were found by univariate analysis $(\mathrm{P}=0.01$, Chi-squared test; $\mathrm{P}=0.013$, Fisher's exact test, respectively) (Table 5). Binomial logistic regression analysis was performed $(\mathrm{P}=0.748$, Hosmer-Lemeshow test) and revealed that metabolic syndrome was significantly associated with salivary occult blood, the number of untreated teeth, and the number of remaining teeth (odds ratio 3.38, $\mathrm{P}=0.041$; odds ratio $1.42, \mathrm{P}=0.01$; and odds ratio $0.84, \mathrm{P}=0.013$, respectively) (Table 6 ).

\section{Discussion}

The usefulness of the SOBT to screen for periodontal disease has been examined in previous research. Basically, salivary hemoglobin can be measured by a colorimetric method or a monoclonal or polyclonal antibody reaction method $[7,8,12-$ 14]. The colorimetric method may not be sufficiently specific for the detection of salivary hemoglobin because of the possibility of contamination by blood from other animals in food [13]. The antibody reaction method is likely to show a higher specificity than the colorimetric method. Several reports suggest that the monoclonal antibody reaction method using paper strips is useful and sufficient for screening for periodontal disease [7, 8, 14]. Additionally, this method is approved for screening for periodontal disease by the Pharmaceutical Affairs Law in Japan. Therefore, we chose this monoclonal antibody reaction method to screen for periodontal disease in this study. Importantly, we excluded individuals who ate or brushed their teeth within $2 \mathrm{~h}$ before the salivary blood test. 
Table 3. Correlation Between Salivary Occult Blood and Factors Associated With General and Oral Health in Workers Aged $\geq 35$ years

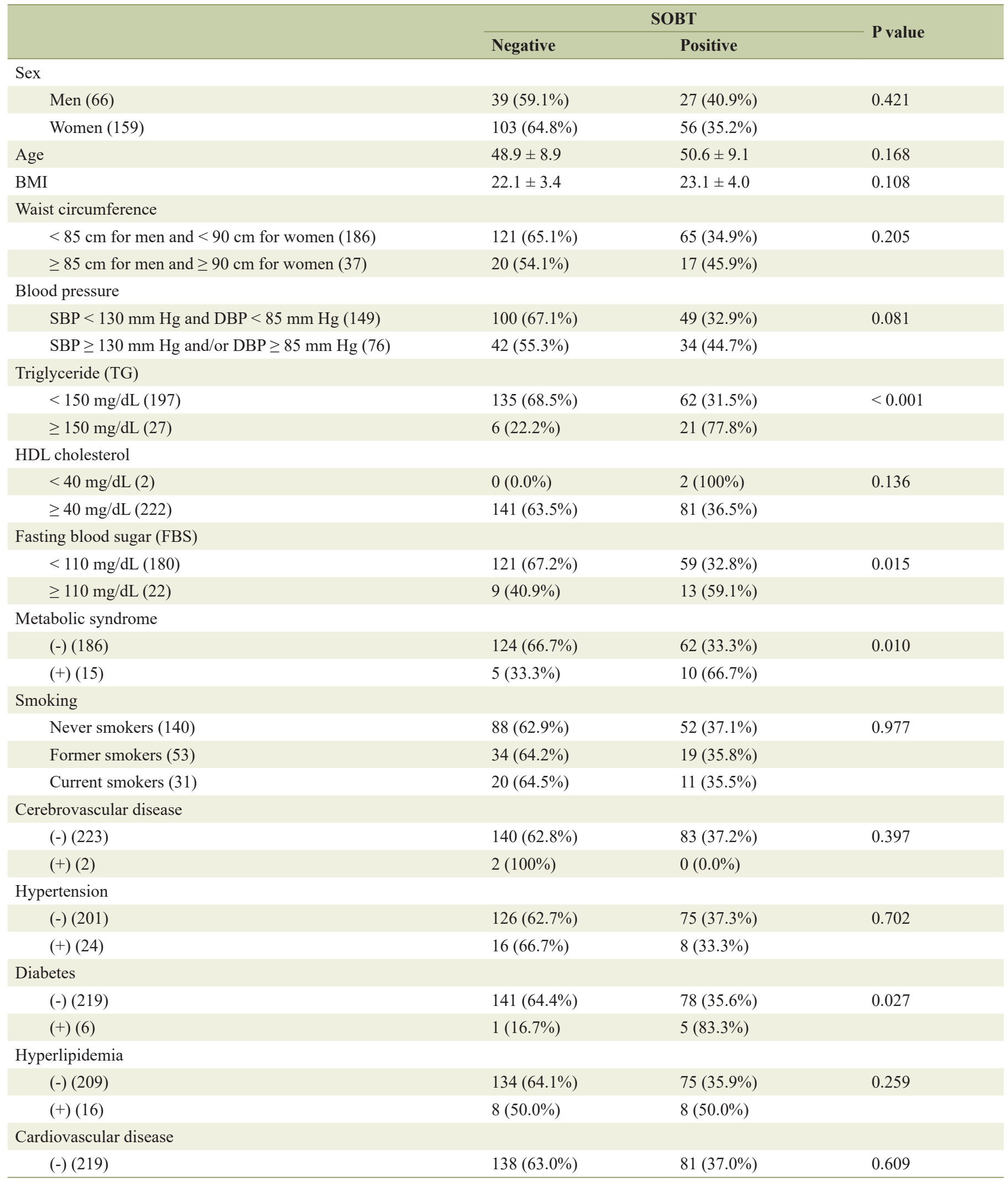


Table 3. Correlation Between Salivary Occult Blood and Factors Associated With General and Oral Health in Workers Aged $\geq 35$ years - (continued)

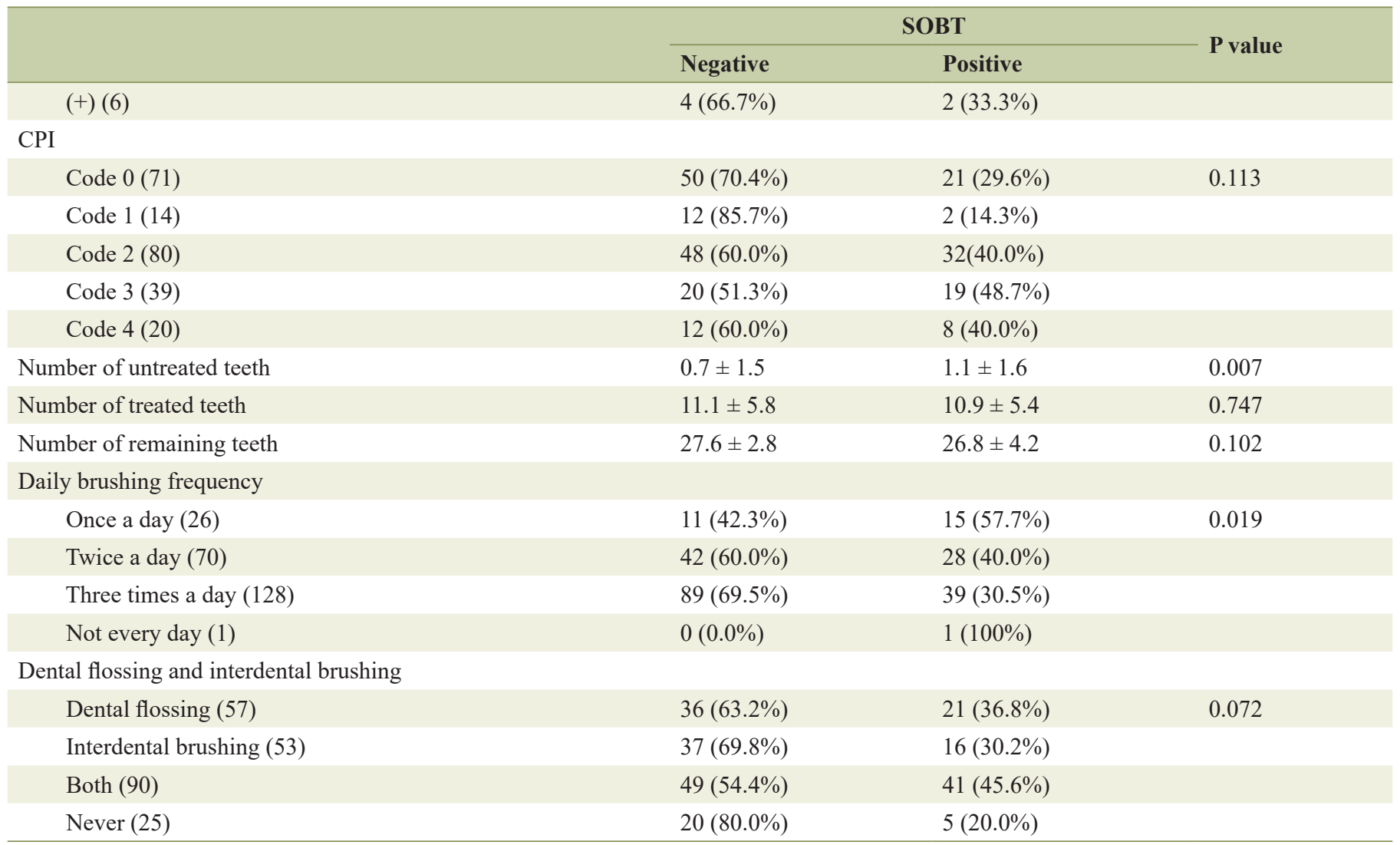

Mann-Whitney U-test, Chi-squared test or Fisher's exact test were used for statistical analysis. P values less than 0.05 were regarded to be statistically significant.

The positive salivary blood test rate was significantly higher in individuals who ate or brushed their teeth within $2 \mathrm{~h}(54.4 \%)$ than in those who did not $(36.2 \%)$. This observation suggests that eating and brushing within the $2 \mathrm{~h}$ before the test can distinctly affect the results of the test.

Statistical analysis revealed that salivary occult blood was significantly associated with CPI. In workers aged $\geq 35$ years, a significant relationship between salivary occult blood and CPI was found by multivariate analysis. These results suggest that salivary occult blood is a reliable screening test for periodontal disease. Furthermore, daily brushing frequency was associated with salivary occult blood in all workers, suggesting that regular oral self-care plays a predominant role in the maintenance of a healthy periodontal condition.
We found a significantly strong positive correlation between salivary occult blood and diabetes in all workers, indicating a close association between periodontitis and diabetes. Periodontal disease influences the prevalence and control of diabetes and diabetes-related complications [15]. Moderate to severe periodontitis is importantly associated with the development of diabetes [16]. Periodontitis-related proinflammatory cytokines (i.e. tumor necrosis factor-alpha (TNF-alpha)) induce systemic inflammation and increase insulin resistance in type 2 diabetic patients [17]. Additionally, adipocyte-produced TNF-alpha is involved in insulin resistance in such patients, suggesting a synergistic effect on insulin resistance by periodontitis and adipocytes [17]. Thus, it is expected that the host inflammatory response plays a vital role in the mecha-

Table 4. Logistic Regression Analysis With Salivary Occult Blood as Dependent Variable in Workers Aged $\geq 35$ Years

\begin{tabular}{llll}
\hline Variables & Odds ratio & $\mathbf{9 5 \%}$ confidence interval & P value \\
\hline Triglyceride (TG) & 7.59 & $2.71-21.20$ & $<0.001$ \\
Diabetes & 3.00 & $0.97-9.26$ & 0.056 \\
CPI & 1.40 & $1.09-1.79$ & 0.007 \\
\hline
\end{tabular}

Binomial logistic regression analysis was conducted using "salivary occult blood" as the dependent variable. P values less than 0.05 were regarded to be statistically significant. 
Table 5. Correlation Between Metabolic Syndrome and Factors Associated With Oral Health

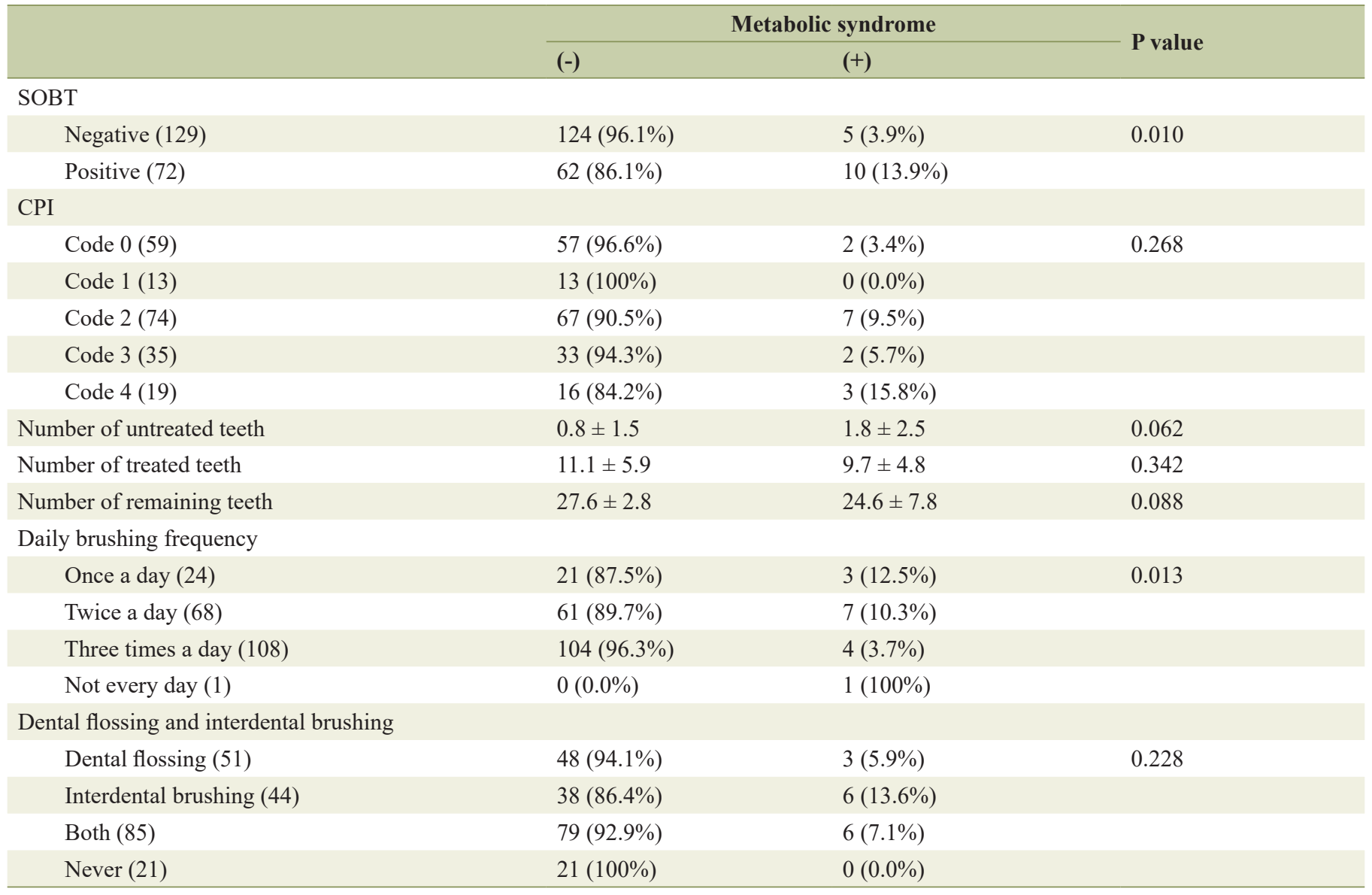

Mann-Whitney $U$ test, Chi-squared test or Fisher's exact test were used for statistical analysis. $P$ values less than 0.05 were regarded to be statistically significant.

nism of periodontal disease and diabetes [18]. Although there was no statistical difference between CPI and diabetes in this study (data not shown), more than half of workers with diabetes recorded a CPI $\geq 1$. This observation is consistent with the previous research that revealed a positive correlation between periodontal disease and diabetes $[15,16]$. To summarize, salivary occult blood may be associated with diabetes as well as periodontal disease. However, it remains unknown whether salivary occult blood reflects the development, prevalence, and deterioration of diabetes.

Metabolic syndrome increases the risk of systemic diseases such as cancer, heart disease and stroke in adults [19-22]. Therefore, it is vital for occupational health services to detect metabolic syndrome at an early stage. Several cross-sectional studies have investigated the correlation between metabolic syndrome and periodontal disease in Japan [23-25]. Metabolic syndrome significantly increased the risk of periodontitis in Japanese people and employees [24]. In this study, high level TGs were identified as a significant factor relating to salivary occult blood in subjects aged $\geq 35$ years. Furthermore, metabolic syndrome was importantly associated with salivary occult blood and the number of untreated teeth in this study. Despite the relatively small number of subjects with metabolic syndrome in this study, our findings suggest that metabolic syndrome might be related to worsened oral health status in Japanese employees. Therefore, regular dental checkups will

Table 6. Logistic Regression Analysis With Metabolic Syndrome as Dependent Variable

\begin{tabular}{llll}
\hline Variables & Odds ratio & $95 \%$ confidence interval & P value \\
\hline Salivary occult blood & 3.38 & $1.05-10.83$ & 0.041 \\
Number of untreated teeth & 1.42 & $1.09-1.85$ & 0.010 \\
Number of remaining teeth & 0.84 & $0.73-0.97$ & 0.013 \\
\hline
\end{tabular}

Binomial logistic regression analysis was conducted using "metabolic syndrome" as the dependent variable. P values less than 0.05 were regarded to be statistically significant. 
be necessary in the workplace, especially in workers with metabolic syndrome.

In conclusion, the SOBT may be a reliable screening tool for periodontal disease. Further investigation is required to clarify the correlations between salivary occult blood and systemic diseases. In the present situation in which it is not common to conduct oral health examinations as a part of occupational health services, regular dental checkups using the SOBT would be helpful in developing and maintaining good oral health associated with general health in Japanese workers.

\section{Acknowledgments}

We thank Mr. Ryotaro Atogami, Ms. Kanae Sumioka, and Ms. Aiko Saito for their kind support throughout this study.

\section{Conflict of Interest}

The authors declare that there is no conflict of interest.

\section{Financial Support}

This study was supported by the Program for Promotion of Periodontal Disease Examination with a Simple Salivary Test.

\section{References}

1. Murray CJ, Lopez AD. Measuring the global burden of disease. N Engl J Med. 2013;369(5):448-457.

2. World Health Organization. Global status report on noncommunicable diseases. Geneva, Switzerland: World Health Organization; 2010.

3. Japan Ministry of Health, Labour and Welfare. Report on the survey of dental diseases. 2016.

4. Noguchi S, Toyokawa S, Miyoshi Y, Suyama Y, Inoue K, Kobayashi Y. Five-year follow-up study of the association between periodontal disease and myocardial infarction among Japanese male workers: MY Health Up Study. J Public Health (Oxf). 2015;37(4):605-611.

5. Simpson TC, Needleman I, Wild SH, Moles DR, Mills EJ. Treatment of periodontal disease for glycaemic control in people with diabetes. Cochrane Database Syst Rev. 2010;5:CD004714.

6. Teeuw WJ, Gerdes VE, Loos BG. Effect of periodontal treatment on glycemic control of diabetic patients: a systematic review and meta-analysis. Diabetes Care. 2010;33(2):421-427.

7. Reed SG, Manz MC, Snipe SM, Ohshima M, Wagner CL. Feasibility study of a salivary occult blood test to correlate with periodontal measures as indicators of periodontal inflammation in a population of pregnant women. J Oral Sci. 2015;57(1):55-58.

8. Shimazaki Y, Akifusa S, Takeshita T, Shibata Y, Doi Y,
Hata J, Ninomiya T, et al. Effectiveness of the salivary occult blood test as a screening method for periodontal status. J Periodontol. 2011;82(4):581-587.

9. [Definition and the diagnostic standard for metabolic syndrome - Committee to Evaluate Diagnostic Standards for Metabolic Syndrome]. Nihon Naika Gakkai Zasshi. 2005;94(4):794-809.

10. World Health Organization. Oral health survey. Basic methods, 4th ed. Geneva, Switzerland: World Health Organization; 1997.

11. Ainamo J, Barmes D, Beagrie G, Cutress T, Martin J, Sardo-Infirri J. Development of the World Health Organization (WHO) community periodontal index of treatment needs (CPITN). Int Dent J. 1982;32(3):281-291.

12. Nam SH, Jung HI, Kang SM, Inaba D, Kwon HK, Kim BI. Validity of screening methods for periodontitis using salivary hemoglobin level and self-report questionnaires in people with disabilities. J Periodontol. 2015;86(4):536545.

13. Nomura Y, Tamaki Y, Tanaka T, Arakawa H, Tsurumoto A, Kirimura K, Sato T, et al. Screening of periodontitis with salivary enzyme tests. J Oral Sci. 2006;48(4):177183.

14. Ohshima M, Zhu L, Yamaguchi Y, Kikuchi M, Nakajima I, Langham CS, Lin W, et al. Comparison of periodontal health status and oral health behavior between Japanese and Chinese dental students. J Oral Sci. 2009;51(2):275281.

15. Graziani F, Gennai S, Solini A, Petrini M. A systematic review and meta-analysis of epidemiologic observational evidence on the effect of periodontitis on diabetes An update of the EFP-AAP review. J Clin Periodontol. 2018;45(2):167-187.

16. Genco RJ, Borgnakke WS. Risk factors for periodontal disease. Periodontol 2000. 2013;62(1):59-94.

17. Nishimura F, Iwamoto Y, Mineshiba J, Shimizu A, Soga Y, Murayama Y. Periodontal disease and diabetes mellitus: the role of tumor necrosis factor-alpha in a 2-way relationship. J Periodontol. 2003;74(1):97-102.

18. Borgnakke WS. Modifiable risk factors for periodontitis and diabetes. Curr Oral Health Rep. 2016;3(3):254-269.

19. Hammarsten J, Damber JE, Haghsheno MA, Mellstrom D, Peeker R. A stage-dependent link between metabolic syndrome components and incident prostate cancer. Nat Rev Urol. 2018;15(5):321-333.

20. Huang PL. A comprehensive definition for metabolic syndrome. Dis Model Mech. 2009;2(5-6):231-237.

21. Roever L, Resende ES, Diniz AL, Penha-Silva N, Veloso FC, Casella-Filho A, Dourado PM, et al. Ectopic adiposopathy and association with cardiovascular disease risk factors: The Uberlandia Heart Study. Int J Cardiol. 2015;190:140-142.

22. van Rooy MJ, Pretorius E. Metabolic syndrome, platelet activation and the development of transient ischemic attack or thromboembolic stroke. Thromb Res. 2015;135(3):434-442.

23. Kushiyama M, Shimazaki Y, Yamashita Y. Relationship between metabolic syndrome and periodontal disease in Japanese adults. J Periodontol. 2009;80(10):1610-1615. 
24. Morita T, Ogawa Y, Takada K, Nishinoue N, Sasaki Y, Motohashi M, Maeno M. Association between periodontal disease and metabolic syndrome. J Public Health Dent. 2009;69(4):248-253.
25. Shimazaki Y, Saito T, Yonemoto K, Kiyohara Y, Iida M, Yamashita Y. Relationship of metabolic syndrome to periodontal disease in Japanese women: the Hisayama Study. J Dent Res. 2007;86(3):271-275. 